\title{
Use of continuous positive airway pressure during flexible bronchoscopy in young children
}

\author{
D. Trachsel*, T.O. Erb" ${ }^{\#}$ F.J. Frei ${ }^{\#}$ and J. Hammer*, on behalf of the Swiss Paediatric \\ Respiratory Research Group
}

ABSTRACT: Young children are at increased risk for hypoxaemia and hypercapnia during flexible bronchoscopy due to the small size and increased collapsibility of their airways. Various strategies are used to prevent hypoventilation and to provide oxygen during the procedure. The aim of this study was to assess the impact of continuous positive airway pressure (CPAP) on ventilation during flexible bronchoscopy in infants and young children.

Tidal breathing was measured in 16 spontaneously breathing and deeply sedated children, aged 3-25 months, by ultrasound spirometry via an airway endoscopy mask. Measurements were made with the tip of the bronchoscope positioned in the pharynx with no CPAP, and in midtrachea with 0,5 and $10 \mathrm{cmH}_{2} \mathrm{O}$ of CPAP.

Transition of the bronchoscope through the vocal cords was associated with significant decreases of tidal volumes $\left(5.0 \pm 0.5\right.$ versus $\left.3.4 \pm 0.5 \mathrm{~mL} \cdot \mathrm{kg}^{-1}\right)$, peak tidal expiratory flows $(78 \pm 12$ versus $\left.52 \pm 10 \mathrm{~mL} \cdot \mathrm{s}^{-1}\right)$ and peak tidal inspiratory flows $\left(98 \pm 15\right.$ versus $\left.66 \pm 12 \mathrm{~mL} \cdot \mathrm{kg}^{-1}\right)$. CPAP (5$10 \mathrm{cmH}_{2} \mathrm{O}$ ) induced almost complete reversal of these changes.

In conclusion, it is shown here that flexible bronchoscopy in spontaneously breathing young children is associated with significant decreases in tidal volume and respiratory flow. These changes are largely reversible with continuous positive airway pressure.

KEYWORDS: Bronchoscopy, continuous positive airway pressure, infant lung function testing, spirometry, ventilation

\begin{abstract}
A dvances in paediatric anaesthesia and technical refinements of the equipment have created opportunities to perform flexible bronchoscopy safely in even the smallest and sickest infants. Improvements include the availability of smaller bronchoscopes and better sedation strategies, using novel short-acting drugs that are rapid in onset and of short duration, allowing excellent patient comfort. A team approach with an anaesthesiologist has now become standard in many paediatric bronchoscopy suites, and is most helpful in small infants and critically ill children. Young children are at increased risk for hypoxaemia and hypercapnia during the procedure due to the small size and increased collapsibility of their airways, and the proportionally much higher resistance imposed by the bronchoscope [1]. Sedatives and anaesthetic drugs utilised to provide comfort during the procedure are also more likely to impair
\end{abstract}

respiratory drive in young children due to the immaturity and sensitivity of the respiratory control system in this age group [2].

This problem has prompted the development of various techniques to make flexible bronchoscopy safer for small children. Strategies included simple efforts to limit the duration of the procedure and the use of supplemental flow-by oxygen or oxygen given through the suction channel [3, 4]. More recently, laryngeal mask airways and specially designed face masks have been used to prevent hypoventilation and to provide oxygen, continuous positive airway pressure (CPAP) or even positive pressure ventilation [5-8].

Previous reports have addressed the alterations of gas exchange occurring during flexible bronchoscopy, primarily with respect to oxygenation [9-12]. However, the changes in tidal
AFFILIATIONS

Division of *Paediatric Intensive Care and Pulmonology, and

\#Paediatric Anaesthesia, University Children's Hospital, Basel,

Switzerland.

CORRESPONDENCE

J. Hammer

Division of Paediatric Intensive Care and Pulmonology

University Children's Hospital of Basel

Post Office Box

4005 Basel

Switzerland

Fax: 41616855004

E-mail: juerg.hammer@unibas.ch

Received:

March 142005

Accepted after revision:

June 142005

SUPPORT STATEMENT

This study was supported by a grant from the Swiss Society of Pulmonology. 
breathing caused by passing a bronchoscope down the airways has never been objectively assessed in infants. The aim of this study was to measure the impact of the bronchoscope and the effect of CPAP on tidal breathing during diagnostic flexible bronchoscopy in infants.

\section{MATERIALS AND METHODS Study subjects}

Sixteen young children in a stable respiratory condition undergoing elective bronchoscopy for a variety of clinical conditions were enrolled consecutively. The study was approved by the Ethics Committee of the University Children's Hospital (Basel, Switzerland) and parental informed consent was obtained.

\section{Study design}

Spirometry measurements were performed in deep sedation with the tip of the flexible bronchoscope positioned in the pharynx without CPAP, and with the tip in mid-trachea at various levels of CPAP, which was applied via sealed face mask and controlled by a pressure gauge in the anaesthesia system.

\section{Methods}

Tidal breathing was measured using an ultrasonic flow sensor (Spiroson®; Eco Medics, Dürnten, Switzerland) attached to an airway endoscopy mask (model 30-30-001; VBM Medizintechnik GmbH, Sulz am Neckar, Germany). The flow sensor's accuracy under varying conditions has been previously validated in ventilated infants [13]. The endoscopy mask consisted of a regular face mask where the original adapter for the fresh gas flow was replaced by a disposable silicon membrane [14]. This setting allows a leak-free passage of a $3.5 \mathrm{~mm}$ flexible bronchoscope (Olympus BF3C20; Olympus Europa GmbH, Hamburg, Germany), which was used for all procedures. An anaesthesia circle system was used to administer $100 \%$ oxygen and CPAP.

All procedures were performed in the Pulmonary Intervention Laboratory, University Children's Hospital, Basel, Switzerland, according to the practice of the University Children's Hospital. Monitoring included three-lead continuous ECG, pulse oximetry and noninvasive blood pressure measurements. Deep sedation was achieved by propofol, generally titrated to a dose of $6-10 \mathrm{mg} \cdot \mathrm{kg}^{-1} \cdot \mathrm{h}^{-1}$ continuous infusion, to reach satisfactory sedation with spontaneous breathing. Adequate time was allowed to achieve a steady state of ventilation before measurements. Topical xylometazoline $0.05 \%$ and $1 \mathrm{~mL}$ of lidocaine $1 \%$ were administered into one nostril during the introduction of sedation.

After application of $0.5 \mathrm{~mL}$ lidocaine $1 \%$ to the vocal cords, measurements of 20-30 consecutive breaths were made with the tip of the bronchoscope located in the hypopharynx without CPAP. The bronchoscope was then advanced into the mid-trachea and measurements were repeated with CPAP of 0,5 and $10 \mathrm{cmH}_{2} \mathrm{O}$. Care was taken to ensure leak-free ventilation at all times. The order at which CPAP was applied was not randomised, in order to exclude any attenuating effect of CPAP on subsequent measurements, allowing the study to be finished within 5-8 min for each patient. This was important to ensure stable conditions in terms of depth of

sedation, which may affect ventilatory parameters in children [15].

The study required the presence of two investigators: one to control the bronchoscope, provide suction and record the readings of the spirometer; the other to maintain the position of the head, to control the face mask and application of CPAP, and to ensure jaw-thrust [16]. The latter investigator was unable to see the display of the spirometry tracings.

\section{Analysis}

Measured parameters included tidal volume, peak tidal expiratory flow, peak tidal inspiratory flow, respiratory rate, and expiratory minute ventilation. Data are presented as mean \pm SE. A repeated-measures ANOVA was used for overall group comparisons of the measurements, the grouping variables being the different study conditions. Bonferroni adjustment was used to determine differences among groups. A p-value $<0.05$ was assigned significance.

\section{RESULTS}

Mean age of the 16 patients was $10 \pm 2$ months, ranging from 3 days to 25 months. Weight distribution was from the 3rd to $>97$ th percentile, with a mean weight of $8.1 \pm 0.8 \mathrm{~kg}$. All patients were in good respiratory condition prior to the procedure. Indications for bronchoscopy and endoscopic findings are listed in table 1.

Significant differences were found in tidal volume $(F=7.27$, $\mathrm{p}<0.0005)$, minute ventilation $(\mathrm{F}=6.26, \mathrm{p}<0.005)$, and peak tidal expiratory and inspiratory flows $(\mathrm{F}=5.83, \mathrm{p}<0.005$; and $\mathrm{F}=9.88, \mathrm{p}<0.0001$, respectively) at different study conditions (table 2). The passage of the flexible bronchoscope through the vocal cords was associated with a significant reduction in tidal volumes and peak tidal flows (Bonferroni p-value $<0.05$ ). Since this was not compensated by increasing respiratory rates within the observation period of 20-30 breaths, the drop in tidal volume resulted in a significant reduction of minute ventilation (Bonferroni p-value $<0.05$ ). Application of 5 and 10 $\mathrm{cmH}_{2} \mathrm{O}$ of CPAP almost fully reversed these negative effects on tidal breathing. A representative flow/time tracing of an individual patient is shown in the figure 1 .

All measurements were well tolerated. None of the subjects demonstrated arterial $\mathrm{O}_{2}$ desaturation by pulse oximetry,

\section{TABLE 1 Indications for bronchoscopy and main findings}

Indication for bronchoscopy

Stridor/upper airway obstruction

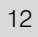

Chronic cough

Bronchoalveolar lavage in cystic fibrosis

Bronchoscopic diagnosis

Laryngomalacia

Chronic bronchitis

Tracheomalacia

Adenotonsillar hyperplasia

Normal airways

3

1

8

Data are presented as $n$ patients. \#: two patients with adenotonsillar hyperplasia had chronic bronchitis. 
TABLE 2 Effect of continuous positive airway pressure (CPAP) on tidal breathing during flexible bronchoscopy

\begin{tabular}{|c|c|c|c|c|}
\hline \multirow[t]{2}{*}{ CPAP $\mathrm{cmH}_{2} \mathrm{O}$} & \multirow{2}{*}{$\frac{\text { Bronchoscope tip in pharynx }}{0}$} & \multicolumn{3}{|c|}{ Bronchoscope tip in trachea } \\
\hline & & 0 & 5 & 10 \\
\hline RR $\min ^{-1}$ & $36 \pm 2$ & $38 \pm 2$ & $36 \pm 2$ & $36 \pm 2$ \\
\hline$V^{\prime} E \mathrm{~mL} \cdot \mathrm{kg}^{-1} \cdot \mathrm{min}^{-1}$ & $176 \pm 17$ & $121 \pm 13^{*}$ & $144 \pm 14$ & $157 \pm 15$ \\
\hline
\end{tabular}

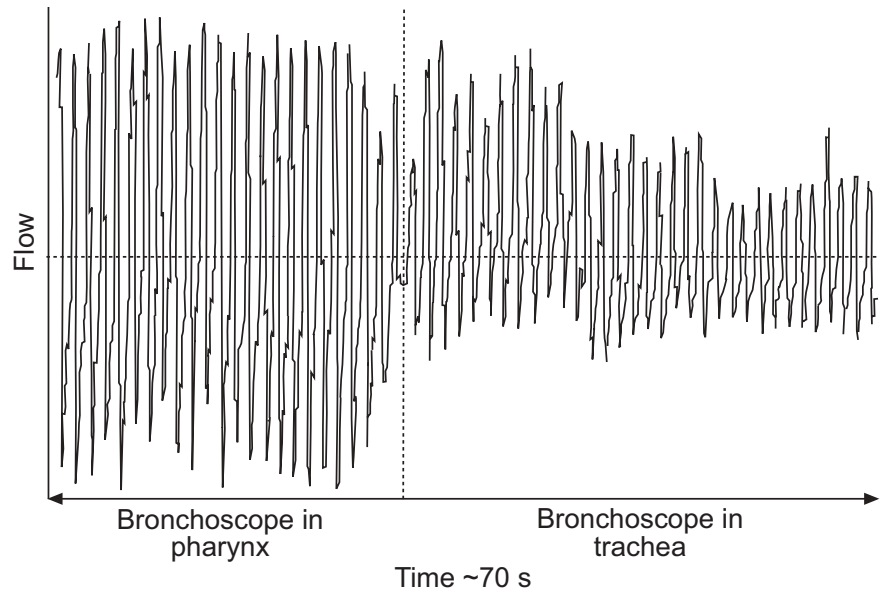

FIGURE 1. Representative flow-time tracing of an individual patient with the bronchoscope positioned above (continuous positive airway pressure (CPAP) 0 $\mathrm{cmH}_{2} \mathrm{O}$; tidal volume (VT) $44 \pm 2 \mathrm{~mL}$ ) and below (CPAP $0 \mathrm{cmH}_{2} \mathrm{O}$; VT $20 \pm 1 \mathrm{~mL}$ ) the vocal cords, respectively

apnoeic episodes, or significant changes in heart rate during the procedure. As arterial partial pressures could not be determined repeatedly, the potential effects of hypoventilation on blood gases could not be demonstrated.

\section{DISCUSSION}

This study is the first to quantify the effect of flexible bronchoscopy on tidal breathing in infants and young children. It demonstrates that passing the bronchoscope into the trachea has a negative impact on tidal breathing and minute ventilation in deeply sedated young children, and that the application of CPAP almost fully restores ventilation to baseline.

Previous studies investigating the effects of flexible bronchoscopy have focused on the temporary alterations of gas exchange occurring during the procedure and the recovery period. The main observation has been the risk of hypoxaemia and hypercapnia caused by alveolar hypoventilation, by an increased ventilation-perfusion mismatch and by augmented metabolic demands, as reflected by increasing cardiac output and oxygen consumption $[1,6,11]$. Studies in patients with acute respiratory failure have shown that CPAP improved oxygenation in this setting, primarily by reducing intrapulmonary shunting and work of breathing [17, 18]. This is likely to be mediated by an increase in functional residual capacity through lung recruitment [19]. The same mechanism has also been proposed as the main reason for the increased tolerance towards flexible bronchoscopy among hypoxaemic adult patients who were assisted with CPAP [6]. However, it seems doubtful that lung recruitment alone would explain the advantageous effects of CPAP during elective bronchoscopy in the infants and children who were in a stable respiratory condition [20].

Increased flexibility of the laryngeal cartilages and the pharyngeal soft tissue predispose the infant to partial or complete upper airway obstruction during tidal breathing [21, 22]. This is even more pronounced in infants with laryngomalacia, which accounts for $\sim 75 \%$ of cases of congenital stridor, as it is the most common cause of symptomatic upper airway obstruction in this age group, and was the main bronchoscopic finding in the present cohort [23]. The cephalad position of the larynx is expected to further increase the proportion of the upper airway resistance to the total airway resistance. Previous work has shown that in deeply sedated infants, adequate ventilation is critically dependent on the flow resistance imposed by the upper airways, and that in this age group $\mathrm{CPAP}$ exerts its beneficial effects on tidal volume primarily through upper airway stenting [16]. It has been shown that CPAP increases the width of the compliant laryngeal opening and decreases the collapsibility of the lateral pharyngeal walls that are thought to be the most compliant structures in the upper airway, thus acting as pneumatic strut [16, 24-26]. In support of this notion, FAUROUX et al. [27] have shown that noninvasive mask ventilation, using positive end-expiratory pressures of 6-8 $\mathrm{cmH}_{2} \mathrm{O}$ and pressure support of 4-10 $\mathrm{cmH}_{2} \mathrm{O}$, was associated with a significant reduction of the work of breathing in infants with laryngomalacia. The observation that ventilation and work of breathing are hardly influenced by CPAP if the upper airways are bypassed by an endotracheal tube provides further evidence of the supraglottic effects of $\mathrm{CPAP}$ during anaesthesia [20]. Another proposed mechanism is that CPAP may make it easier to overcome the increased intrinsic positive end-expiratory pressure created by the bronchoscope obstructing the airway. Thus, improved upper airway patency and counterbalancing of the increased intrinsic 
positive end-expiratory pressure may both facilitate tidal breathing with CPAP during flexible bronchoscopy.

The relatively short duration of measurements and the fact that blood gases could not be measured are recognised limitations of this study. Hence, it could not be shown if the changes in ventilation were paralleled by changes in arterial partial pressures of $\mathrm{CO}_{2}$ and $\mathrm{O}_{2}$. This study centred on the impact of CPAP during short periods, because this best demonstrates a causal relationship between intervention and effect, ensures stable conditions in terms of depth of sedation and complies with the ethical guidelines, which do not allow unnecessarily prolonged procedures. The authors would not have expected carbon dioxide arterial tension to change dramatically within the relatively short observation periods of 20-30 spontaneous breaths. Nevertheless, it seems reasonable to assume that continued hypoventilation would lead to significant changes of blood gases in those susceptible to hypoxaemia and hypercapnia.

This study was intended to describe the vulnerability of tidal breathing during flexible bronchoscopy in very young children and to assess the benefits of adding CPAP via an endoscopy mask. It is possible that the drop in minute ventilation observed after passing the bronchoscope through the vocal cords might be different with other sedation regimens that cause less respiratory depression, e.g. halothane or ketamine [15]. Nevertheless, the results have clinical implications, because propofol has become a popular and frequently used agent for sedation during endoscopies at many places [4, 28, 29]. In addition, the size of the bronchoscope may also affect the observed alterations in tidal breathing. Hence, the current authors recommend using the smallest instrument available to minimise the effect of instrumental airway obstruction on tidal breathing.

The application of CPAP effectively improved ventilatory parameters during flexible bronchoscopy in these young children. However, it is important to realise that CPAP may hinder the visual detection of dynamic airway lesions by preventing airway collapse. Dynamic airway lesions both in the extra- and intrathoracic airways need to be studied without CPAP to assess their collapsibility. The beauty of the current technique, however, is that it allows the investigator to study such lesions with and without CPAP and to visualise the benefit of CPAP application.

In conclusion, the passage of a flexible bronchoscope through the vocal cords causes a significant reduction of tidal volumes and minute ventilation in infants and young children. The administration of continuous positive airway pressure through an endoscopy mask improves these variables during flexible bronchoscopy in deeply sedated infants, thereby providing further safety and time for the examination.

\section{REFERENCES}

1 Schnapf BM. Oxygen desaturation during fiberoptic bronchoscopy in pediatric patients. Chest 1991; 99: 591-594.

2 Motoyama EK. Respiratory physiology in infants and children. In: Motoyama EK, Davis PJ, eds. Smith's Anesthesia for Infants and Children. St. Louis, MosbyYear Book, Inc., 1996; pp. 11-67.
3 Ernst A, Silvestri GA, Johnstone D. Interventional pulmonary procedures: guidelines from the American College of Chest Physicians. Chest 2003; 123: 1693-1717.

4 Midulla F, de Blic J, Barbato A, et al. Flexible endoscopy of paediatric airways. Eur Respir J 2003; 22: 698-708.

5 Antonelli M, Conti G, Rocco M, et al. Noninvasive positivepressure ventilation vs conventional oxygen supplementation in hypoxemic patients undergoing diagnostic bronchoscopy. Chest 2002; 121: 1149-1154.

6 Maitre B, Jaber S, Maggiore SM, et al. Continuous positive airway pressure during fiberoptic bronchoscopy in hypoxemic patients. Am J Respir Crit Care Med 2000; 162: 1063-1067.

7 Nussbaum E, Zagnoev M. Pediatric fiberoptic bronchoscopy with a laryngeal mask airway. Chest 2001; 120: 614-616.

8 Smyth AR, Bowhay AR, Heaf LJ, Smyth RL. The laryngeal mask airway in fibreoptic bronchoscopy. Arch Dis Child 1996; 75: 344-345.

9 Albertini RE, Harrell JHI, Kurihara N, Moser KM. Arterial hypoxemia induced by fiberoptic bronchoscopy. JAMA 1974; 230: 1666-1667.

10 Franchi LM, Maggi JC, Nussbaum E. Continuous end-tidal $\mathrm{CO}_{2}$ in pediatric bronchoscopy. Pediatr Pulmonol 1993; 16: 153-157.

11 Peerless JR. Continuous intraarterial blood gas monitoring during flexible fiberoptic bronchoscopy in critically ill patients. J Bronchol 1996; 3: 262-265.

12 Erb T, Hammer J, Rutishauser M, Frei FJ. Fiberoptic bronchoscopy in sedated infants facilitated by an airway endoscopy mask. Paediatr Anaesth 1999; 9: 47-52.

13 Scalfaro P, Cotting J, Sly PD. In vitro assessment of an ultrasonic flowmeter for use in ventilated infants. Eur Respir J 2000; 15: 566-569.

14 Frei FJ, Wengen D, Rutishauser M, Ummenhofer W. The airway endoscopy mask: useful device for fibreoptic evaluation and intubation of the paediatric airway. Paediatr Anaesth 1995; 5: 319-324.

15 Kulkarni P, Brown KA. Ventilatory parameters in children during propofol anaesthesia: a comparison with halothane. Can J Anaesth 1996; 43: 653-659.

16 Hammer J, Reber A, Trachsel D, Frei FJ. Effect of jaw-thrust and continuous positive airway pressure on tidal breathing in deeply sedated infants. J Pediatr 2001; 138: 826-830.

17 Schlobohm RM, Falltrick RT, Quan SF, Katz JA. Lung volumes, mechanics, and oxygenation during spontaneous positive-pressure ventilation: the advantage of CPAP over EPAP. Anesthesiology 1981; 55: 416-422.

18 Katz JA, Marks JD. Inspiratory work with and without continuous positive airway pressure in patients with acute respiratory failure. Anesthesiology 1985; 63: 589-607.

19 Sivan Y, Deakers TW, Newth CJ. Effect of positive endexpiratory pressure on respiratory compliance in children with acute respiratory failure. Pediatr Pulmonol 1991; 11: 103-107.

20 Keidan I, Fine GF, Kagawa T, Schneck FX, Motoyama EK. Work of breathing during spontaneous ventilation in anesthetized children: a comparative study among the face mask, laryngeal mask airway and endotracheal tube. Anesth Analg 2000; 91: 1381-1388. 
21 Mathew OP, Roberts JL, Thach BT. Pharyngeal airway obstruction in preterm infants during mixed and obstructive apnea. J Pediatr 1982; 100: 964-968.

22 Miller MJ, Carlo WA, DiFiore JM, Martin RJ. Airway obstruction during periodic breathing in premature infants. J Appl Physiol 1988; 64: 2496-2500.

23 Holinger LD. Etiology of stridor in the neonate and child. Ann Otol Rhinol Laryngol 1980; 89: 323-399.

24 Gaon P, Lee S, Hannan S, Ingram D, Milner AD. Assessment of effect of nasal continuous positive pressure on laryngeal opening using fibre optic laryngoscopy. Arch Dis Child Fetal Neonatal Ed 1999; 80: F230-F232.

25 Kuna ST, Bedi DG, Ryckman C. Effect of nasal airway positive pressure on upper airway size and configuration. Am Rev Respir Dis 1988; 138: 969-975.
26 Schwab RJ, Pack AI, Gupta KB, et al. Upper airway and soft tissue structural changes induced by CPAP in normal subjects. Am J Respir Crit Care Med 1996; 154: 1106-1116.

27 Fauroux B, Pigeot J, Polkey MI, et al. Chronic stridor caused by laryngomalacia: work of breathing and effects of noninvasive ventilatory assistance. Am J Respir Crit Care Med 2001; 164: 1874-1878.

28 Berkenbosch JW, Graff GR, Stark JM, Ner Z, Tobias JD. Use of remifentanil-propofol mixture for pediatric flexible fiberoptic bronchoscopy sedation. Paediatr Anaesth 2004; 14: 941-946.

29 Zestos MM, Bhattacharya D, Rajan S, Kemper S, Haupert M. Propofol decreases waste anesthetic gas exposure during pediatric bronchoscopy. Laryngoscope 2004; 114: 212-215. 